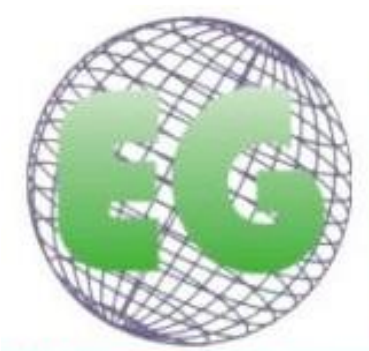

ISSN 1695-6141 $N^{\circ} 49$

\title{
Modelo de Credibilidad Emprendedora en los estudiantes de enfermería y fisioterapia
}

The Entrepreneurial Credibility Model on students from nursing and physiotherapy

\author{
Marc Mussons-Torras ${ }^{1}$ \\ Elisenda Tarrats-Pons ${ }^{2}$ \\ ${ }^{1}$ Doctor por la UPC. Universidad de Vic - Universidad Central de Cataluña.España. \\ ${ }^{2}$ Doctora por la UPC. Profesora Agregada y Directora del Departamento de la Facultad de Empresa y \\ Comunicación. Universidad de Vic - Universidad Central de Cataluña.España.
}

E-mail: marc.mussons@uvic.cat

http://dx.doi.org/10.6018/eglobal.17.1.280281

Recibido: $14 / 01 / 2017$

Aceptado: 22/04/2017

\section{RESUMEN:}

Objetivo: El artículo analiza cuáles son los factores sociodemográficos, rasgos de personalidad y variables endógenas y exógenas de viabilidad que ejercen una mayor influencia en la conducta del estudiante emprendedor de los grados universitarios de enfermería y fisioterapia.

Método: El esquema teórico se basa en el modelo del potencial emprendedor de Krueger y Brazeal, desarrollado a partir de los modelos del evento emprendedor de Shapero y Sokol, y de la teoría de la conducta planificada de Ajzen.

El estudio identifica las variables clave en la percepción de deseabilidad y viabilidad que afectan directamente a la credibilidad emprendedora del estudiante. La muestra está formada por 302 estudiantes de grados de ciencias de la salud (122 de enfermería y 180 de fisioterapia).

Resultados: La ecuación del modelo de credibilidad emprendedora determina la gran incidencia de las variables de percepción de viabilidad, especialmente de la autoeficacia y de la creatividad e innovación. El modelo incluye también las variables de esfuerzo y perseverancia, desempleo y financiación.

Conclusiones: Los programas formativos orientados al desarrollo de la competencia del emprendimiento serán efectivas, si inciden en factores de personalidad como apertura, propensión al riesgo, responsabilidad y motivación del logro, pues todas ellas inciden directamente en la variable clave de creatividad e innovación.

El estudio también destaca que los estudiantes de fisioterapia son más emprendedores que los estudiantes de enfermería.

Palabras clave: Credibilidad Emprendedora; Autoeficacia; Creatividad e Innovación; Esfuerzo y Perseverancia; Desempleo; Financiación.

\section{ABSTRACT:}

Objective: This paper analyses social and demographic factors, personality traits and the endogenous and exogenous variables of feasibility in order to find out the ones that generate the greatest influence on the entrepreneurial behaviour from physiotherapy and nursing students. 
Method: The study is based on the Krueger and Brazeal model of entrepreneurial potential, which is a mix of the Shapero's model of the entrepreneurial event, and the Ajzen's theory of planned behaviour.

The research identifies the key variables of perceived desirability and feasibility on the entrepreneur student credibility. The data were collected from 302 students of health science degrees: nursing (122); physiotherapy (180).

Results: The equation model of the credibility on entrepreneurship shows the importance of the feasibility perception, especially on innovation and creativity, and self- efficacy. The model also includes the variables of effort and perseverance, unemployment and funding.

Conclusion: The educative programs on entrepreneurship are effective when they impact to personality traits as openness, propensity to risk, responsibility and need of achievement. This is because each one of them affects directly to the variable key of creativity and innovation.

The study confirms that physiotherapy students are more likely to become entrepreneurs than nursing students are.

Keywords: Entrepreneurship Credibility; Self-Efficacy; Creativity and Innovation; Effort and Perseverance; Unemployment; Funding

\section{INTRODUCCIÓN}

La Declaración de Bolonia ${ }^{(1)}$ establece un nuevo marco universitario para que los titulados puedan hacer frente a la demanda de un mercado laboral cada vez más globalizado y exigente donde la investigación orientada en la innovación deberá ser clave para asegurar un crecimiento económico inteligente y sostenible de la Unión Europea.

La Unión por la innovación engloba directrices y políticas de la Comisión Europea para salir de la recesión, centradas en promover el espíritu emprendedor, apoyando a jóvenes empresas innovadoras, y reforzando los vínculos entre educación, empresa, investigación e innovación. La Comisión Europea, en la estrategia Europa 2020, y en línea con el tratado de Bolonia y la estrategia de Lisboa, refuerza la cooperación entre universidad y empresa, mediante la construcción de puentes de $\mathrm{I}+\mathrm{D}+\mathrm{i}$, que fomente un crecimiento inteligente, basado en la innovación y el conocimiento.

Uno de los aspectos fundamentales para que la universidad se adapte con éxito a la nueva sociedad del conocimiento es que ésta promueva y adopte nuevos roles y actividades lejos de sus tradicionales funciones de formación. Entre ellos destacamos el fomento del espíritu emprendedor, la transferencia del conocimiento, la formación continua y la innovación, así como la creación de vínculos cada vez más fuertes entre universidad y empresa que deberán servir para que la universidad lidere nuevas transformaciones de éxito que supongan un mayor impacto económico, social y cultural $^{(2)}$.

En este contexto, analizar la competencia emprendedora de nuestros estudiantes universitarios será clave, dado que los futuros empresarios deberán de innovar a partir del conocimiento.

El eje dinamizador de la nueva economía tiene su origen en el modelo de la triple hélice de Etzkowitz ${ }^{(3)}$, que establece lazos entre la universidad, la empresa y el sector público. La Comisión Europea adopta dicho modelo y lo dota de fondos específicos llamados RIS3 (Research Innovation Smart Specialisation Strategies). Es por ello, que el acto de emprender, y el emprendimiento en general, se convierten en un elemento esencial y estratégico. 
El elemento clave de todo el proceso de emprender es la intención que implica que la decisión de emprender es voluntaria y consciente ${ }^{(4)}$. Y es el mejor predictor del futuro comportamiento emprendedor, que lo antecede ${ }^{(5,6)}$.

El análisis de la intención emprendedora es determinante para poder explicar la creación de empresas. Muchos autores han desarrollado investigaciones empíricas tratando de obtener los determinantes que inciden en el sujeto a través del enfoque cognitivo.

Los modelos de emprendimiento tienen su origen en el enfoque cognitivo, que interpreta que cualquier cosa que hacemos o decimos como seres humanos es influenciado directamente por procesos mentales, tales como la motivación, las percepciones o las actitudes (7). Por esta razón, los primeros estudios sobre emprendimiento se centraban en la motivación, que es una de las principales razones por las que los individuos deciden crear un nuevo negocio (8). Los modelos de intención emprendedora, según el enfoque cognitivo, parten de la teoría sobre el aprendizaje social, que destaca la influencia del entorno y de la sociedad en el emprendimiento $^{(9)}$. Es también de dicho autor, la definición de autoeficacia como la percepción de control en el desempeño profesional, que como veremos más adelante, es un elemento clave en los resultados de nuestro estudio.

Otro de los modelos de intención emprendedora es el modelo del evento emprendedor de Shapero y Sokol ${ }^{(10)}$. De acuerdo con este modelo, la elección del sujeto para iniciar una nueva empresa depende de tres conjuntos de elementos: la percepción de deseabilidad, la percepción de viabilidad y la propensión a actuar. Donde la percepción de viabilidad se refiere a la habilidad consciente del individuo para desempeñar con éxito un comportamiento emprendedor, equivalente al concepto de autoeficacia de Bandura.

Según Shapero ${ }^{(10)}$, el sujeto siempre elegirá un comportamiento que sea creíble. Es decir, la opción creíble, surge por combinación positiva entre el deseo y la viabilidad. Y solamente el comportamiento es creíble, si el sujeto lo percibe como deseable y viable.

Estas percepciones son las que explican en gran medida la intención emprendedora del sujeto, aunque siempre en un nivel potencial. Pues dicho potencial necesita de eventos significativos y cruciales en la vida del sujeto, para que finalmente el sujeto decida emprender. Shapero ${ }^{(10)}$ ofrece en su estudio multitud de ejemplos de empresas que son fundadas en circunstancias especiales, como cuando el emprendedor pierde su anterior empleo, recibe un premio de lotería, o le sucede cualquier otro cambio personal que puede accionar el suceso disparador y convertir el potencial emprendedor en una intención real de emprender.

Por otro lado, el modelo de Ajzen ${ }^{(5)}$ sobre intención emprendedora se basa en la teoría de la conducta planificada, donde su fundamento principal se construye bajo la premisa de que el comportamiento humano requiere de cierta cantidad de planificación precedente, y que en dicha planificación, así como en su comportamiento posterior, le influyen las creencias. Dichas creencias afectan directamente a las intenciones que son planificadas en base a dos factores: por un lado, a la actitud hacia la acción. Y por otro lado, a las normas subjetivas. Ambos factores se refieren a la deseabilidad de la conducta, y equivaldrían al concepto de percepción de deseabilidad de los autores Shapero y Sokol ${ }^{(10)}$. Además de dichos 
factores referentes a la deseabilidad, Ajzen ${ }^{(5)}$ distingue también un tercer factor llamado control conductual percibido. Dicho factor se refiere a la factibilidad de la conducta, que equivaldría al concepto de percepción de viabilidad de Shapero y Sokol ${ }^{(10)}$ y al concepto de autoeficacia de Bandura ${ }^{(11)}$.

Finalmente, Krueger y Brazeal ${ }^{(12)}$ complementan el modelo de Shapero y Sokol ${ }^{(10)}$, incorporando aspectos de Ajzen, y lo denominan modelo del potencial emprendedor, donde se integran todos los conceptos de percepción de viabilidad y deseabilidad, así como la propensión a actuar y el suceso disparador. Es importante destacar que el modelo del potencial emprendedor (figura 1), surge de la evolución de los anteriores modelos, siendo en la actualidad el modelo de emprendimiento más utilizado. De hecho, es la base teórica de nuestra investigación.

En este modelo se destaca la relación de las percepciones de deseabilidad y de viabilidad como factores determinantes de la credibilidad emprendedora.

Se define la percepción de deseabilidad como lo más o menos atractivo que resulta para la persona el hecho de poner en marcha un negocio. Por lo tanto, estas actitudes personales derivarán en posibles resultados, más o menos favorables, atribuibles a la acción. Y sobre esta creencia de posibles resultados, influirán factores que van desde la propia personalidad del sujeto, hasta las normas sociales percibidas, entendidas como la percepción que tiene el individuo de su entorno social, y acerca de lo que piensan en positivo o en negativo sobre la posibilidad de crear una nueva empresa.

Por otro lado, se define la percepción de viabilidad como el grado en que la persona se siente capaz de llevar a cabo la iniciativa emprendedora. Siendo ésta una sensación derivada de la propia percepción de control interno, pero también de la percepción de la propia capacidad, de saberse en posesión de los recursos financieros necesarios, de tener la información adecuada, de verse capaz de superar los riesgos, de mantener la incertidumbre bajo control, y de entender que el nuevo proyecto empresarial es una buena oportunidad de negocio. En definitiva, el sujeto es autoeficaz, y se asegura con antelación suficiente la viabilidad de la creación de su nueva empresa. Entendiendo a la autoeficacia como aquella capacidad cognitiva del individuo que le permite sentirse capaz de saber y conseguir movilizar los recursos necesarios para poder controlar cualquier situación que se le presente a lo largo de su vida $^{(11)}$.

Un efecto importante de la percepción de autoeficacia en el individuo es la selección de su comportamiento. Los individuos suelen evitar situaciones que a priori no controlan. $Y$ al revés, los individuos buscan aquellas situaciones donde consideran que pueden controlar mejor ${ }^{(11)}$. Y lo que es más importante, las personas escogen su carrera profesional en función de lo que se consideran correctamente competentes y preparados. Lo cual, podemos deducir, al menos dentro del marco teórico que si somos capaces de preparar a los futuros estudiantes en la creación de su futuro negocio, incrementaremos también la intención hacia el emprendimiento. Y esto es especialmente relevante para los estudiantes de fisioterapia, que como veremos están mucho más orientados a la creación de su propio negocio. 
Figura n¹: Modelo teórico del potencial emprendedor ${ }^{(12)}$

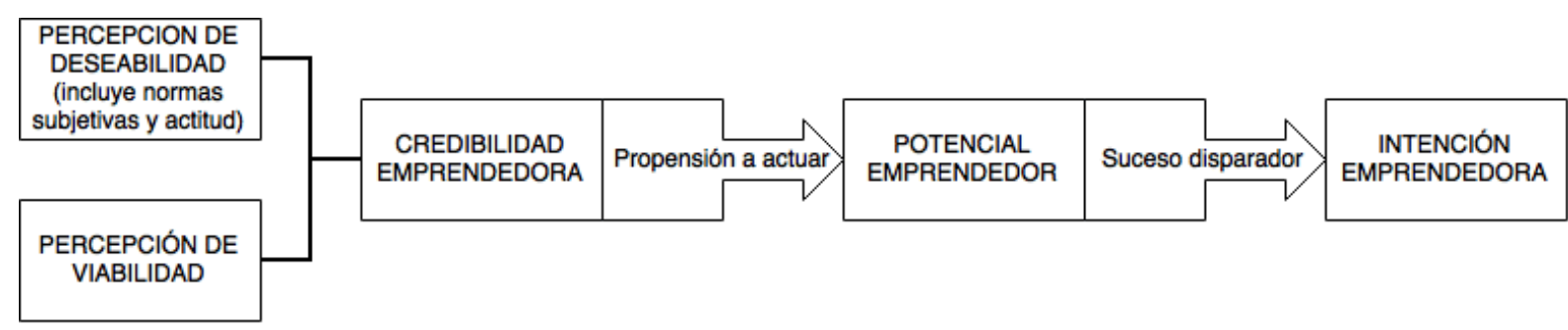

\section{MÉTODO}

La investigación pretende encontrar el modelo de credibilidad emprendedora de los estudiantes de los grados universitarios de enfermería y fisioterapia, mediante las siguientes fases:

Fase de prueba piloto: para verificar la validez de contenido y de constructo del instrumento/formulario y su fiabilidad.

Fase definitiva: elaboración de la ecuación de regresión múltiple del modelo de credibilidad emprendedora, y el contraste de la hipótesis nula (Ho: Los estudiantes de enfermería en relación a los estudiantes de fisioterapia tienen un nivel similar de credibilidad emprendedora).

Para el tratamiento de los datos se ha utilizado el software estadístico SPSS en su versión 22.0.0.

\section{Fase prueba piloto}

El cuestionario se ha construido ad-hoc, utilizando ítems procedentes de las investigaciones científicas de los autores más relevantes.

La variable dependiente, denominada credibilidad emprendedora, está formada por ítems basados en la escala de actitudes de orientación emprendedora de los autores Robinson, Stimpson y Huefner ${ }^{(13)}$, siendo asimismo utilizados por Singh y DeNoble (14) (ítems no7,9 y 11).

Las variables independientes se agrupan en las dimensiones de percepción de deseabilidad y de percepción de viabilidad.

En la percepción de deseabilidad se incluyen los factores sociodemográficos (edad, género, antecedentes familiares, experiencia laboral previa y formación en cursos de empresa) y los factores de personalidad (neuroticismo, extraversión, apertura, amabilidad, responsabilidad, autoestima, automonitoreo, propensión al riesgo, motivación del logro y locus control).

En la percepción de viabilidad se incluyen los factores económicos de tipo exógeno (ciclo económico, financiación y desempleo) y los factores personales o de tipo endógeno (creatividad e innovación, esfuerzo y perseverancia, autoeficacia). 


\section{Validez de contenido}

La validez de contenido determina si el instrumento de medida, sirve para medir el comportamiento que se pretende medir. Es por este motivo, que en la construcción de las dimensiones se han utilizado ítems que previamente han sido contrastados y utilizados por otros autores de referencia. Los ítems fueron revisados por un juicio de expertos de la Universidad de Vic - Universidad Central de Cataluña. Según los autores Skjong y Wentwortht ${ }^{(15)}$, los criterios que un experto debe poseer son experiencia en la realización de juicios, y expertise en la temática objeto de análisis. También es determinante su grado de objetividad, de imparcialidad y de buena reputación en la comunidad.

Los resultados del juicio de expertos indicaron que la mayoría de los ítems eran adecuados y bien redactados. Solamente en algunos ítems de nueva creación era necesaria alguna corrección, especialmente de comprensión y simplificación por uso de frases con doble negativo, por ejemplo.

\section{Validez de constructo}

Para determinar la validez de constructo y los índices de fiabilidad del instrumento, se realizó la prueba piloto con una muestra de 84 estudiantes. El método estadístico utilizado es aleatorio por conglomerados de forma natural, donde la unidad muestral es un grupo de elementos de la población que forman una unidad (en concreto la jornada del programa de emprendimiento Aracoop Empren en clau Coop, que se desarrolló en la UVIC-UCC en septiembre de 2015).

Los datos obtenidos para cada dimensión fueron analizados factorialmente mediante la extracción de componentes principales con rotación Varimax. En cada conjunto de ítems, que explican una variable o dimensión, se determina si todos los ítems correlacionan adecuadamente con su dimensión.

Como criterio general, George y Mallery ${ }^{(16)}$ consideran coeficientes fiables los alfa de cronbach superiores a 5 . Siendo el rango de 5 a 6 , suficiente (pero pobre). En nuestro caso hay una variable por debajo de 5 que es esfuerzo y perseverancia. Esto nos indicaría que el análisis de los estudiantes para este factor debe hacerse con cautela y evaluando los resultados sabiendo que pueden ser poco fiables. Para el resto, y una vez eliminada la variable propensión a actuar, nos indicaría que tenemos un cuestionario fiable y cuyos resultados pueden ser analizados asumiendo la validez de contenido y de constructo del formulario.

\section{Fase definitiva}

La muestra está formada por 302 estudiantes de los grados de enfermería $(40,4 \%)$ y fisioterapia (59,6\%), obtenida en 2015 , de los cuales el $88 \%$ son menores de 25 años, y el $63 \%$ son mujeres. Además, el 55\% tienen antecedentes familiares empresarios, el $69 \%$ disponen de experiencia laboral previa, y un 23\% han cursado alguna formación en creación de empresas.

La técnica estadística para hallar la ecuación que nos permite medir y predecir la credibilidad emprendedora de los estudiantes de los grados de salud es la regresión lineal múltiple, y una vez garantizados los siguientes supuestos: 
- Linealidad: En nuestro estudio se rechaza la hipótesis nula de que los valores no tienen relación lineal con la variable dependiente $(F=69,648$; prob 0,000).

- Normalidad: Se cumple, pues la regresión del residuo tipificado en relación a la variable dependiente traza un gráfico de distribución normal (figura 2), así como el alineamiento en diagonal del residuo tipificado (figura 3).

Figura no2: Histograma del residuo estandarizado

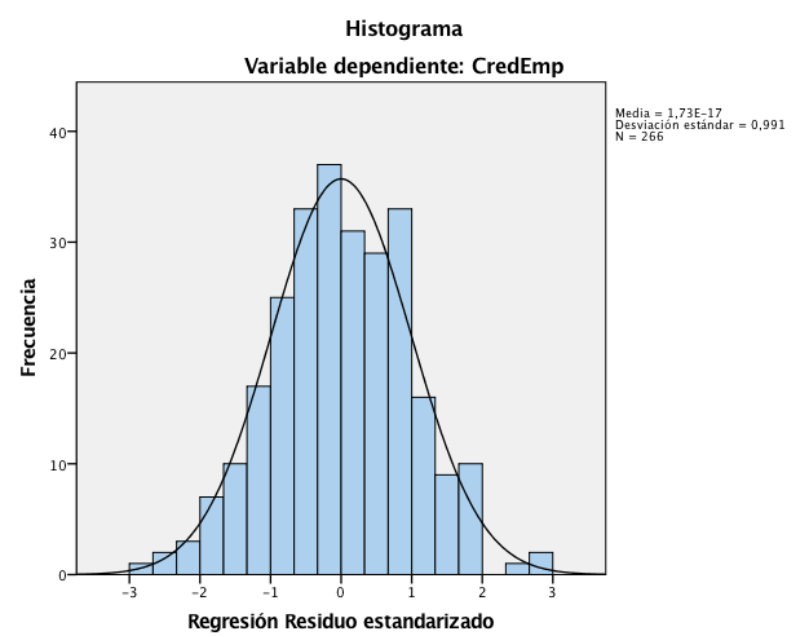

Figura no3: Regresión residuo estandarizado

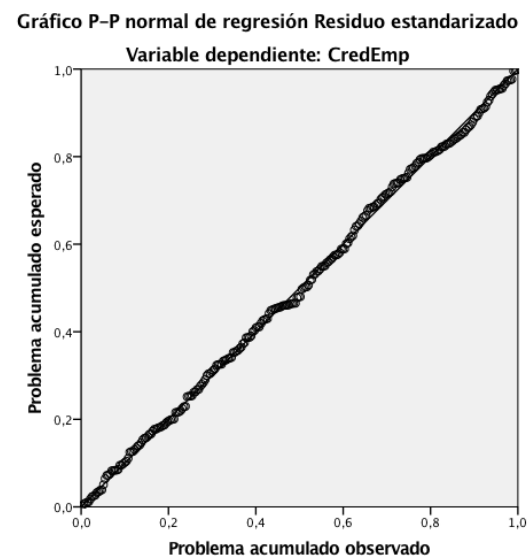

- Independencia de los residuos: Se cumple si son aleatorios e independientes entre sí, siendo el estadístico Durbin Watson cercano a $2(=1,833)$.

- Homocedasticidad: La varianza de los residuos es constante para cada una de las combinaciones de las variables independientes en relación a la dependiente. Se cumple observando que hay nube de puntos y no correlación entre los residuos tipificados y los valores pronosticados tipificados (figura 4). 
Figura no4: Diagrama de dispersión del residuo estandarizado

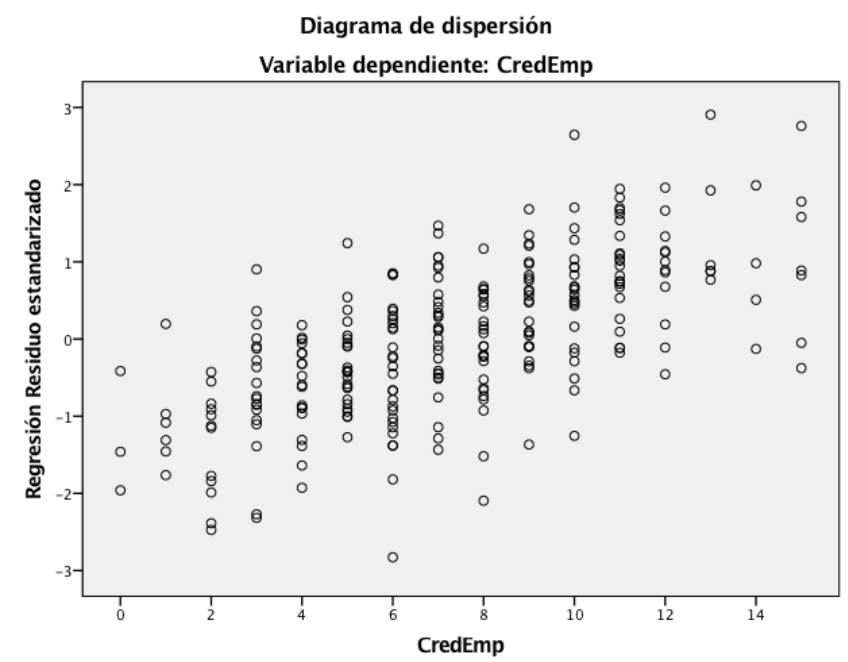

- No colinealidad: Se cumple si no existe relación lineal entre ninguna de las variables independientes. En nuestro caso, todos los valores de tolerancia están entre ,704 y ,848; y los factores de inflación de la varianza (FIV) entre 1,309 y 1,420 (tabla 1).

Tabla no1: Estadísticas de colinealidad del modelo

\begin{tabular}{|l|r|r|}
\hline Variables & Tolerancia & VIF \\
\hline Autoeficacia & 0,704 & 1,42 \\
\hline Creatividad e innovación & 0,848 & 1,18 \\
\hline Esfuerzo y perseverancia & 0,718 & 1,393 \\
\hline Desempleo & 0,747 & 1,339 \\
\hline Financiación & 0,764 & 1,309 \\
\hline
\end{tabular}

VIF: factor de inflación de la varianza; variable dependiente: credibilidad emprendedora

La investigación utiliza la técnica de la regresión múltiple por pasos sucesivos, introduciendo las variables con mayor beta y más significativas, para luego observar los cambios en el $\mathrm{R}$ cuadrado del modelo.

Consideraremos necesaria la variable introducida siempre que se cumplan las siguientes condiciones:

*La variación en $\mathrm{R}$ cuadrado sea superior a 0,01.

${ }^{*}$ El índice de condición se sitúe en los entornos de 10-20, indicando poca colinealidad $^{(17)}$.

${ }^{*}$ El factor de inflación de la varianza (FIV) sea inferior a 10 en todas las variables ${ }^{(18)}$

En la tabla ํ2 se observa que a medida que incorporamos variables, la explicación de la varianza se incrementa. Es importante destacar la elevada importancia que adopta la primera variable incorporada. La autoeficacia nos explica el $40,2 \%$ de la variación de toda la varianza de la variable dependiente de credibilidad emprendedora. 
Los requisitos de no colinealidad del índice de condición y de la FIV se cumplen. Para las cinco dimensiones del modelo, el índice de condición se sitúa en los 12,944 (tabla 3 ), y con sólo dos combinaciones de variables con una proporción de varianza más elevada (tabla 4). Mientras que la FIV de las variables no supera los 1,42 (tabla1).

Tabla n2: Construcción del modelo de credibilidad emprendedora

\begin{tabular}{|r|l|r|r|r|r|}
\hline Modelos & $\mathbf{R}$ & $\begin{array}{l}\mathbf{R} \\
\text { cuadrado }\end{array}$ & $\begin{array}{l}\text { R } \\
\text { cuadrado } \\
\text { ajustado }\end{array}$ & $\begin{array}{l}\text { Error estándar } \\
\text { de la } \\
\text { estimación }\end{array}$ & $\begin{array}{l}\text { Cambio en R } \\
\text { cuadrado }\end{array}$ \\
\hline 1 &, 634 & 0,402 & 0,4 & 2,645 & 0,402 \\
\hline 2 &, 695 & 0,483 & 0,479 & 2,465 & 0,08 \\
\hline 3 &, 730 & 0,532 & 0,527 & 2,349 & 0,049 \\
\hline 4 &, 750 & 0,563 & 0,556 & 2,276 & 0,03 \\
\hline 5 &, 757 & 0,573 & 0,564 & 2,255 & 0,01 \\
\hline
\end{tabular}

Modelos: 1: constante + autoeficacia; 2: constante + autoeficacia + creatividad e innovación; 3: constante + autoeficacia + creatividad e innovación + esfuerzo y perseverancia; 4: constante + autoeficacia + creatividad e innovación + esfuerzo y perseverancia + desempleo; 5: constante + autoeficacia + creatividad e innovación + esfuerzo y perseverancia + desempleo + financiación.

Tabla no3: Diagnósticos de colinealidad

\begin{tabular}{|r|r|r|}
\hline Dimensión & Autovalor & Índice de condición \\
\hline & & 1 \\
\hline 1 & 5,36 & 3,982 \\
\hline 2 & 0,338 & 7,256 \\
\hline 3 & 0,102 & 7,415 \\
\hline 4 & 0,097 & 8,731 \\
\hline 5 & 0,07 & 12,944 \\
\hline 6 & 0,032 & \\
\hline
\end{tabular}

Variable dependiente: credibilidad emprendedora

Tabla n-4: Proporciones de varianza

\begin{tabular}{|r|r|r|r|r|r|}
\hline Constante & Autoeficacia & $\begin{array}{l}\text { Creatividad e } \\
\text { innovación }\end{array}$ & $\begin{array}{l}\text { Esfuerzo y } \\
\text { perseverancia }\end{array}$ & Desempleo & Financiación \\
\hline 0 & 0 & 0 & 0 & 0,01 & 0 \\
\hline 0,01 & 0 & 0,01 & 0,01 & 0,79 & 0 \\
\hline 0,01 & 0,61 & 0,09 & 0,11 & 0,15 & 0,13 \\
\hline 0,02 & 0,28 & 0 & 0,23 & 0 & 0,52 \\
\hline 0,1 & 0,09 & 0,14 & 0,62 & 0,05 & 0,27 \\
\hline 0,86 & 0,01 & 0,76 & 0,03 & 0,01 & 0,08 \\
\hline
\end{tabular}

Variable dependiente: credibilidad emprendedora

\section{RESULTADOS}

La ecuación resultante del modelo de credibilidad emprendedora es (tabla 5):

Credibilidad Emprendedora $=-1,75+0,53$ Autoeficacia $+0,43$ Creatividad e innovación + 0,41 Esfuerzo y perseverancia + 0,37 Desempleo + 0,18 Financiación 
Tabla n5: Ecuación del modelo de credibilidad emprendedora

\begin{tabular}{|r|l|r|l|}
\hline Modelos & Variables & Beta & Error estándar \\
\hline 1 & Constante & 2,48 & 0,40 \\
\hline & Autoeficacia & 0,85 & 0,06 \\
\hline 2 & Constante & $-0,73$ & 0,63 \\
\hline & Autoeficacia & 0,78 & 0,06 \\
\hline & Creatividad e innovación & 0,61 & 0,10 \\
\hline 3 & Constante & $-1,23$ & 0,60 \\
\hline & Autoeficacia & 0,66 & 0,06 \\
\hline & Creatividad e innovación & 0,43 & 0,10 \\
\hline & Esfuerzo y perseverancia & 0,49 & 0,09 \\
\hline 4 & Constante & $-1,22$ & 0,58 \\
\hline & Autoeficacia & 0,57 & 0,06 \\
\hline & Creatividad e innovación & 0,44 & 0,09 \\
\hline & Esfuerzo y perseverancia & 0,43 & 0,09 \\
\hline & Desempleo & 0,44 & 0,10 \\
\hline 5 & Constante & $-1,75$ & 0,62 \\
\hline & Autoeficacia & 0,53 & 0,07 \\
\hline & Creatividad e innovación & 0,43 & 0,09 \\
\hline & Esfuerzo y perseverancia & 0,41 & 0,09 \\
\hline & Desempleo & 0,37 & 0,11 \\
\hline & Financiación & 0,18 & 0,07 \\
\hline
\end{tabular}

Coeficientes no estandarizados; variable dependiente: credibilidad emprendedora.

El modelo de credibilidad emprendedora del presente estudio obtiene un elevado índice de $\mathrm{R}$ cuadrado. La bondad de ajuste del modelo se sitúa en los ,571. Esto significa que las variables independientes explican el $57 \%$ del total de la varianza de credibilidad emprendedora.

Este dato es significativo, especialmente si lo comparamos con los valores de ajuste de otros modelos de emprendimiento (tabla 6).

Tabla no6: Valores de ajuste de otros modelos

\begin{tabular}{|l|l|}
\hline Autor & Bondad de ajuste (R cuadrado) \\
\hline (Brice, 2002) & 0,07 \\
\hline (Singh \& DeNoble, 2003) & 0,18 \\
\hline (Crant, 1996) & 0,3 \\
\hline (Korunka, Frank, Lueger, \& Mugler, 2003) & 0,29 \\
\hline $\begin{array}{l}\text { (Lounsbury, Gibson, Sundstrom, Wilburn \& } \\
\text { Loveland, 2004) }\end{array}$ & 0,42 \\
\hline (Naldi, Nordqvist, Sjöberg, \& Wiklund, 2007) & 0,29 \\
\hline (Lee \& Tsang, 2001) & 0,32 \\
\hline
\end{tabular}

En nuestra investigación se rechaza la hipótesis nula de que los estudiantes de enfermería en relación a los estudiantes de fisioterapia tienen un nivel similar de credibilidad emprendedora (nivel de significación 0,00). El estudio nos indica que el colectivo analizado de estudiantes de fisioterapia tiene un comportamiento emprendedor distinto al colectivo de estudiantes de enfermería (tabla ํㅡㄱ). 
Tabla n-7: Contraste de hipótesis

\begin{tabular}{|l|r|}
\hline U de Mann-Whitney & 5872 \\
\hline W de Wilcoxon & 13253 \\
\hline$Z$ & $-6,484$ \\
\hline Sig. asintótica (bilateral) & 0 \\
\hline
\end{tabular}

Además, si observamos los datos estadísticos descriptivos (tabla ํo8), podemos afirmar que el colectivo de estudiantes de fisioterapia es más emprendedor. En concreto, su media es de 8,31, y su desviación estándar de 2,993. Todo lo contrario que los estudiantes de enfermería, que obtienen una media más baja $(5,91)$, y una desviación estándar más elevada (indicando mayor dispersión de los resultados).

Tabla n-8: Estadísticos descriptivos por grado

\begin{tabular}{|c|c|c|c|}
\hline Grados & & Estadístico & $\begin{array}{l}\text { Error } \\
\text { estándar }\end{array}$ \\
\hline \multirow[t]{10}{*}{ Enfermería } & Media & 5,91 & 0,306 \\
\hline & $\begin{array}{l}95 \% \text { de intervalo de } \\
\text { confianza para la } \\
\text { media }\end{array}$ & $5,3-6,5$ & \\
\hline & Media recortada al 5\% & 5,73 & \\
\hline & Mediana & 5 & \\
\hline & Varianza & 11,367 & \\
\hline & Desviación estándar & 3,371 & \\
\hline & Mínimo & 0 & \\
\hline & Máximo & 15 & \\
\hline & Rango & 15 & \\
\hline & Rango intercuartil & 4 & \\
\hline \multirow[t]{10}{*}{ Fisioterapia } & Media & 8,31 & 0,227 \\
\hline & $\begin{array}{l}\text { 95\% de intervalo de } \\
\text { confianza para la } \\
\text { media }\end{array}$ & $7,86-8,76$ & \\
\hline & Media recortada al 5\% & 8,34 & \\
\hline & Mediana & 8 & \\
\hline & Varianza & 8,955 & \\
\hline & Desviación estándar & 2,993 & \\
\hline & Mínimo & 0 & \\
\hline & Máximo & 15 & \\
\hline & Rango & 15 & \\
\hline & Rango intercuartil & 4 & \\
\hline
\end{tabular}

\section{DISCUSIÓN}

La ecuación del modelo está formada por variables que inciden en la credibilidad emprendedora del estudiante universitario. Siendo la variable autoeficacia, la percepción de control en el comportamiento ${ }^{(5)}$. Para el sujeto representa su percepción de viabilidad en la intención a emprender ${ }^{(10,19)}$, siendo el mejor predictor al buen desempeño profesional ${ }^{(11)}$, reduciendo errores, contratiempos y problemas que puedan surgir durante el emprendimiento ${ }^{(9)}$. La autoeficacia es una variable 
fuertemente significativa en relación al potencial emprendedor en los estudios realizados con estudiantes universitarios ${ }^{(4,20,22)}$.

La variable creatividad e innovación se define como el estado de alerta hacia nuevas oportunidades. El sujeto creativo e innovador es capaz de detectar nuevas oportunidades, que han pasado desapercibidas por el resto. Para Athayde (23), las personas creativas son los percusores del comportamiento innovador. La correlación entre innovación y emprendimiento es significativa, siendo especialmente fuerte como percepción de viabilidad ${ }^{(13,24,25)}$. En nuestro estudio, la variable obtiene una Beta (=.43) elevada, que indica su influencia directa con la credibilidad emprendedora del sujeto. Este dato significativo lo podemos complementar analizando el efecto de correlación con otras variables de percepción de deseabilidad respecto a la creatividad e innovación (tabla $\mathrm{n}-9$ ). En este sentido, la motivación del logro (Rho=.526) se define como el deseo del sujeto por mejorar los resultados de la acción, y sentirse responsable de los mismos. Se trata de un deseo personal de competición hacia el máximo nivel de excelencia, buscando la mejor manera de hacer el trabajo, y siendo algo muy importante para el sujeto. En una línea parecida encontramos la variable responsabilidad ( $\mathrm{Rho}=.393)$, concepto que tiene relación en ser diligente, bien organizado, persistente y orientado a objetivos y resultados ${ }^{(14)}$. Y la variable apertura $(\mathrm{Rho}=.380)$, siendo las personas abiertas tendentes a ser más creativas, originales y receptivas a nuevas experiencias; especialmente en distintos y variados intereses ${ }^{(14)}$. Es de hecho la originalidad y la creatividad, los factores que más correlacionan con el emprendimiento, porque son el preludio de la innovación ${ }^{(23)}$.

Tabla n9: Correlaciones de variables con creatividad e innovación (Rho de spearman)

\begin{tabular}{|l|l|l|l|l|}
\hline $\begin{array}{l}\text { Creatividad e } \\
\text { innovación }\end{array}$ & Apertura & Responsabilidad & $\begin{array}{l}\text { Motivación del } \\
\text { logro }\end{array}$ & $\begin{array}{l}\text { Propensión } \\
\text { al riesgo }\end{array}$ \\
\hline $\begin{array}{l}\text { Coeficiente de } \\
\text { correlación }\end{array}$ &, $380^{* *}$ &, $393^{* *}$ &, $526^{* *}$ &, $388^{* *}$ \\
\hline Sig. (bilateral) & 0 & 0 & 0 & 0 \\
\hline $\mathrm{N}$ & 267 & 271 & 295 & 291 \\
\hline
\end{tabular}

La variable propensión al riesgo se define como la preferencia por las situaciones que pueden aportar beneficios y recompensas en caso de éxito, pero también severas consecuencias en caso de fracaso ${ }^{(26)}$.

La variable esfuerzo y perseverancia, se relaciona con el coste de oportunidad, y va más allá del puro sacrificio en tiempo y dinero. En realidad, emprender supone la renuncia a un trabajo más estable ${ }^{(14)}$. Pero también tiene implicaciones de sacrificio a nivel económico. El emprendedor debe comprometer recursos financieros, de forma periódica, y de acuerdo con su proyecto de negocio ${ }^{(27)}$. En los estudios con alumnos universitarios, se constató la variable del esfuerzo como factor clave en el emprendimiento ${ }^{(14,28)}$.

En relación a la variable desempleo, la teoría de la marginación de Veciana ${ }^{(29)}$, entendida como la propensión a convertirse en empresarios aquellas personas con escasas oportunidades laborales, constata la relación existente entre desempleo y emprendimiento por necesidad ${ }^{(21,28)}$. Esta correlación positiva se constata en nuestro 
modelo, donde mayores niveles de desempleo, generan mayor credibilidad emprendedora, aunque sea por necesidad y autoempleo.

La variable financiación, y en concreto la falta de financiación y acceso al crédito, se percibe por el sujeto como un freno y un obstáculo en su percepción de viabilidad del proyecto emprendedor ${ }^{(28,30)}$. Los informes anuales del Global Entrepreneurship Monitor afirman que en España hay un incremento de las dificultades de financiación, con una menor aportación de capital semilla a la actividad emprendedora y con una mayor necesidad de aportación de financiación ajena, especialmente desde 2008.

\section{CONCLUSIONES}

A lo largo de la investigación hemos analizado los distintos factores que influyen en la posibilidad de que un estudiante del ámbito de salud acabe siendo emprendedor.

Nuestro punto de partida a nivel teórico lo hemos enmarcado en el modelo del potencial emprendedor de los autores Krueger y Brazeal ${ }^{(12)}$, que como hemos visto, incorpora la fusión de los dos grandes modelos de emprendimiento de Shapero y Sokol ${ }^{(10)}$ y Ajzen ${ }^{(5)}$, cuyos orígenes se remontan en los años ochenta y noventa respectivamente.

El modelo de Krueger y Brazeal ${ }^{(12)}$ establece la posibilidad de evaluar a los estudiantes en su potencial emprendedor, siendo la aparición del denominado "suceso disparador", el preludio a la intención emprendedora.

Es importante destacar que el potencial emprendedor incorpora la propensión a actuar, que posibilita que una conducta emprendedora creíble, acabe siendo factible en todo su potencial.

Sin embargo, nuestra investigación se ha centrado en el análisis de las variables que de alguna forma pueden incidir, ya sea a nivel socioeconómico, a nivel formativo y/o a nivel político, en la credibilidad emprendedora.

O lo que es lo mismo, la combinación de percepciones de deseabilidad y de viabilidad al emprendimiento. Siendo nuestro modelo capaz de detectar la combinación de factores necesarios para que un estudiante tenga un comportamiento emprendedor creíble.

El modelo de credibilidad emprendedora propuesto define la conducta del estudiante universitario emprendedor como creíble cuando el sujeto es creativo e innovador, capaz de detectar nuevas oportunidades de negocio, sabiendo lo que tiene que hacer, siendo perseverante y dispuesto a esforzarse en tiempo y dinero. $Y$ todo ello contextualizado por factores externos que influyen directamente en su percepción de viabilidad como son niveles altos de desempleo y accesibilidad al crédito.

Asimismo, el modelo final tiene otras aportaciones interesantes, como conocer que hay factores que inciden directamente sobre la variable de creatividad e innovación. Es por ello que si queremos estudiantes universitarios más emprendedores, debemos de potenciar su creatividad e innovación, y a todas las variables que directamente correlacionan con ésta; como son la apertura del sujeto, la responsabilidad, la tolerancia al riesgo, la motivación por el éxito y por los resultados obtenidos. 
Es interesante observar que la variable creatividad e innovación es ubicada dentro del conjunto de las variables de viabilidad, definida como "la búsqueda de nuevas oportunidades de mercado". Pero en cambio, le inciden directamente variables del entorno de deseabilidad, moldeables por la personalidad del sujeto y, por lo tanto, que pueden ser modificadas e incrementadas a través de los programas y planes de estudio de los grados de fisioterapia y enfermería.

La conclusión final del estudio nos indica claramente que son más bien pocas las variables que realmente inciden en un futuro comportamiento emprendedor, teniendo mayor peso las relativas a la percepción de viabilidad, y especialmente a las relacionadas con la autoeficacia, y con la innovación y la creatividad.

Finalmente, también es significativa la conclusión de que los estudiantes de fisioterapia son más emprendedores que los estudiantes de enfermería. Aunque los dos colectivos son formados dentro de un mismo ámbito de conocimiento, la profesión ejercida es en términos generales muy distinta una vez finalizados sus estudios universitarios.

\section{REFERENCIAS}

1. Eurostat. Eurostat statistical book. Publications_Office, editor. European Union; 2009.

2. Reichert S. Institutional diversity in European higher education. Zurich: European University Association; 2009.

3. Etzkowitz H, Leydesdorff L. The triple helix as a model for innovation studies. Sci public policy. 1998;

4. Krueger NF, Reilly MD, Carsrud AL. Competing models of ntrepreneurial intentions. J Bus Ventur. 2000;15(5-6):411-32.

5. Ajzen I. The theory of planned behavior. Organ Behav Hum Decis Process. $1991 ; 50: 179-211$.

6. Kolvereid L. Prediction of employment status choice intentions. Entrep Theory Pract. 1996;21(1):47-57.

7. Krueger NF. The impact of prior entrepreneurial exposure on perceptions of new venture feasibility and desirability. Entrep Theory Pract.1993;18(1):5-21.

8. Shane S, Locke EA, Collins CJ. Entrepreneurial motivation. Hum Resour Manag Rev. 2003;257-279.

9. Bandura A. The Social Foundations of Thought and Action. Prentice-Hall, editor. Englewood Cliffs, NJ.; 1986.

10. Shapero A, Sokol L. The social dimension of entrepreneurship. Prentice H. Kent $\mathrm{CA}$, Sexton DL, Vesper $\mathrm{KH}$, editors. The encyclopedia of entrepreneurship.Englewood Cliffs, NJ.; 1982. 72-90 p.

11.Wood R, Bandura A. Social cognitive theory of organizational management. Acad Manag Rev. 1989;14:361-84.

12. Krueger Jr NF, Brazeal D V. Entrepreneurial Potential and Potential Entrepreneurs. Entrepreneurship Theory and Practice. 1994;91-104.

13. Robinson PB, Stimpson D V, Huefner JC, Hunt HK. An attitude approach to the prediction of entrepreneurship. Entrep Theory Pract. 991;15(4):13-31.

14. Singh G, DeNoble A. Views on self-employment and personality: an exploratory study. J Dev Entrep. 2003;8(3):265-81.

15. Skjong R, Wentworth B. Expert Judgement and risk perception. Det Nor Verit. 2000;

16. George D, Mallery P. SPSS for Windows step by step: a Simple Guide and Reference. 4th ed. Bacon A\&, editor. Boston; 2003. 
17. Belsley DA. Conditioning diagnostics: collinearity and weak data in regression. In: John Wiley \& Sons I, editor. 1991.

18. Neter J, Wasserman W, Kutner MH. Applied Linear Statistical Models. M.A: Irwin. 1990.

19. Krueger NF, Carsrud AL. Entrepreneurial intentions: applying the theory of planned behavior. Entrep Reg Dev. 1993;5(4):315-30.

20. Chen CC, Greene PG, Crick A. Does entrepreneurial self-efficacy distinguish entrepreneurs from managers? J Bus Ventur. 1998;13:295-316.

21. Linan F, Urbano D, Guerrero M. Regional variations in entrepreneurial cognitions: Start-up intentions of university students in Spain. Entrep Reg Dev. $2011 ; 23(3-4)$.

22. Liñán F, Chen Y-W. Development and Cross-Cultural Application of a Specific Instrument to Measure Entrepeneurial Intentions. Entrep Theory Pract. 2009;593-617.

23. Athayde R. Measuring Enterprise Potential in Young People. Entrep Theory Pract. 2009;481-500.

24. Ismail $\mathrm{N}$, Jaffar $\mathrm{N}$, Siow $\mathrm{T}$. Using EAO model to predict the self-employment intentions among the Universities' Undergraduates in Malaysia. Int $\mathrm{J}$ Trade, Econ Financ. 2013;4:282-7.

25. Wang W, Lu W, Millington JK. Determinants of Entrepreneurial Intention among

College Students in China and USA. J Glob Entrep Res. 2011;1(1):35-44.

26. Brockhaus $\mathrm{RH}$. Risk taking propensity of entrepreneurs. Acad Manag J. 1980;23(3):509-20.

27. Hisrich R, Peters M. Entrepreneurship. Vol. 5. Nueva York: Mc Graw Hill; 2002.

28. Espí MT, Arana G, Heras I, Diaz A. Perfil emprendedor del alumnado universitario del campus de Gipuzkoa de la Upv/Ehu. Rev Dir y Adm Empres. 2007;14:83-110.

29. Veciana JM. Creación de empresas como programa de investigación científica. Rev Eur Dir y Econ la Empres. 1999;8(3):11-36.

30. Lüthje C, Franke N. The "making" of an entrepreneur: testing a model of entrepreneurial intent among engineering students at MIT. R\&D Manag. 2003;33(2):135-47.

ISSN 1695-6141

(C) COPYRIGHT Servicio de Publicaciones - Universidad de Murcia 